\title{
Surface Reflectance Model Estimation from Daylight Illuminated Image Sequences
}

\author{
Robert Love and Nicholas D Efford \\ School of Computer Studies \\ The University of Leeds \\ Leeds, LS2 9JT \\ United Kingdom \\ $\{$ rcl,nde\}@scs.leeds.ac.uk
}

\begin{abstract}
Accurate surface reflection models derived from existing natural scenes can be used for a variety of tasks. This paper presents a machine vision approach for determining such models. We investigate the use of simplistic models of reflection and daylight illumination to determine surface reflection properties. We attempt to determine the gloss factor of both an individual surface and a multi-faceted object when illuminated by natural light with varying sun position. Experiments are performed using synthetic image sequences of surfaces illuminated by CIE standard clear and intermediate skies, viewed from a variety of camera positions. Results show that some success can be achieved using such simple illumination models. Enhancements to the proposed method are also discussed with a view to improving system performance.
\end{abstract}

\section{Introduction}

Object information obtained from outdoor scenes using a single static camera can be used in a wide variety of applications. In this paper we focus on the determination of surface reflection properties. Such information can be used, along with shape information, for tasks such as object recognition, remote surface inspection and virtual environment building based on existing scenes. The need for increasingly realistic virtual environments based upon existing scenes requires detailed knowledge of how light interacts with the constituent surfaces. Typically virtual world builders resort to reflectance tables compiled for known surfaces or estimate surface properties. It would be desirable to have an automated method for determining such properties. Gagalowicz [4] has recently highlighted the need for such methods in the domain of telepresence systems.

In this paper we present our work on determining the surface reflectance of scenes illuminated solely by daylight. We assume that the scene is viewed over an extended period of time using a single static camera. Figure 1 shows a typical seven hour sequence viewing a building illuminated by a clear sky. It can clearly be seen that over this time period the apparent brightness of surface's varies between each frame of the sequence. This variation is due to the changing position and luminance of the sun and sky. This paper shows that by discarding sky irradiance and using sun position alone the time of peak apparent surface 
brightness can be used to determine surface reflection properties. We develop methods using local isotropic reflection models to predict when this peak luminance will occur for various surface types. Though of modest accuracy, the results show that some success can be achieved using such simplistic models of daylight illumination and local reflection. Although these results have been obtained from the study of synthetic images we expect our methods to perform similarly when presented with real images. Results from real images have not been presented in this paper as the algorithms are still in the early stages of development. The use of synthetic images allows system testing using images of known scenes which are free from effects such as noise, lens abberation, and camera response.
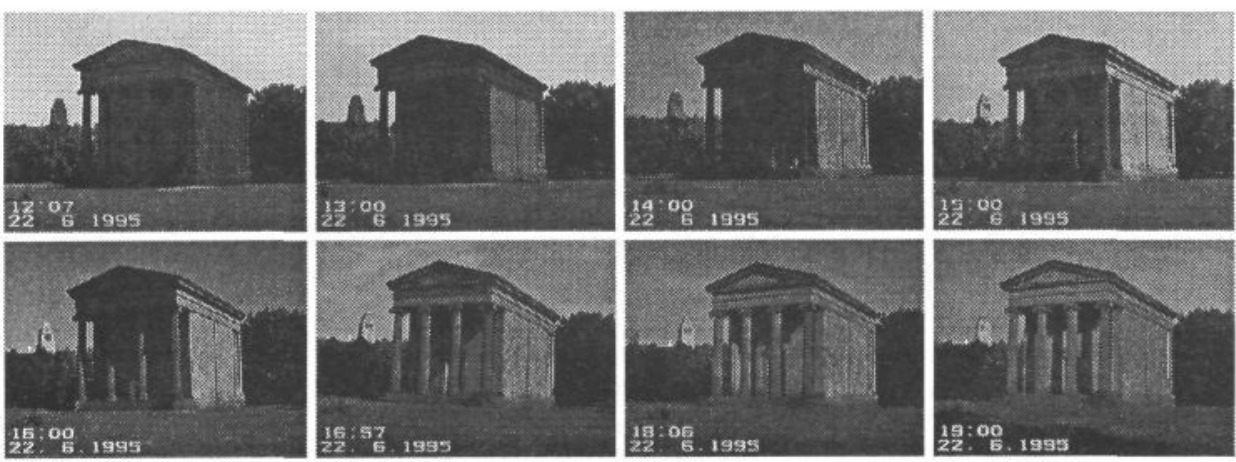

Figure 1: Image sequence showing frames taken hourly between 12:00 and 19:00 of a building illuminated by clear sky viewed from the northwest.

Nayer et al have performed similar work using a number of extended sources placed around a surface sample [7]. Their photometric sampling technique attempts to determine both surface orientation and reflection properties. Although we assume a priori knowledge of surface orientation, we extend their work by considering the sun as the moving illuminant. This work represents a significant move away from the study of laboratory scenes. Much of the work conducted in this field of computer vision has focused on simple scenes viewed in favourable lighting conditions. In this paper we develop an approach suitable for real scenes viewed in natural light.

\section{Surface Reflection}

The radiance of a differential surface element is related to the incident irradiance by the bidirectional reflection distribution function (BRDF) [8]. The BRDF, $f_{r}\left(\vec{\omega}_{i} \rightarrow \vec{\omega}_{r}\right)$, determines the fraction of incident irradiance from a differential solid angle $\vec{\omega}_{i}$ reflected in a direction $\vec{\omega}_{r}$. In this way the BRDF can be used to fully describe reflection from all different types of surface, including anisotropic surfaces, assuming Helmholtz reciprocity.

Given the incident and reflected radiance, $L_{i}$ and $L_{r}$, and their related directions it is possible to determine the BRDF for that particular instance of the geometry:

$$
f_{r}\left(\vec{\omega}_{i} \rightarrow \vec{\omega}_{r}\right)=\frac{L_{r}\left(\vec{\omega}_{r}\right)}{L_{i}\left(\vec{\omega}_{i}\right) \cos \theta_{i} d \omega_{i}} . \quad\left[s r^{-1}\right]
$$




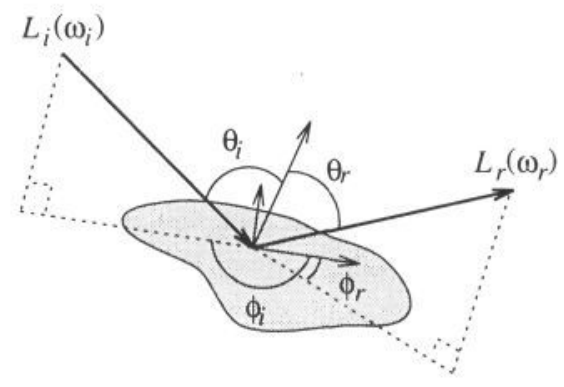

Figure 2: Surface reflection geometry.

The total quantity of light reflected in the direction $\vec{\omega}_{r}$ is due to the light incident from a hemisphere of directions $\Omega_{i}$. Since the BRDF is a linear function this can be calculated as

$$
L_{r}\left(\vec{\omega}_{r}\right)=\int_{\Omega_{i}} f_{r}\left(\vec{\omega}_{i} \rightarrow \vec{\omega}_{r}\right) L_{i}\left(\vec{\omega}_{i}\right) \cos \theta_{i} d \omega_{i} .
$$

This is referred to as the reflectance equation [1].

It is usually easier to work in terms of reflectance than BRDF as this is constrained to lie between 0 and 1 . Reflectance represents the ratio of the reflected flux to the incident flux and is therefore dimensionless [8]. Reflectance can be defined in terms of BRDF [1]:

$$
\rho\left(\vec{\omega}_{i} \rightarrow \vec{\omega}_{r}\right)=\frac{\int_{\Omega_{r}} \int_{\Omega_{i}} f_{r}\left(\vec{\omega}_{i} \rightarrow \vec{\omega}_{r}\right) \cos \theta_{i} d \omega_{i} \cos \theta_{r} d \omega_{r}}{\int_{\Omega_{i}} \cos \theta_{i} d \omega_{i}} .
$$

For computer vision and graphics purposes the reflectance function is typically modelled as being the sum of diffuse, mirror and glossy components. For the purposes of this paper, the mirror component is ignored as it is rarely significant in natural scenes. By using an independent reflectance function for each component a composite model can be derived:

$$
\rho\left(\vec{\omega}_{i} \rightarrow \vec{\omega}_{r}\right)=(1-\gamma) \cdot \rho_{\text {diffuse }}\left(\vec{\omega}_{i} \rightarrow \vec{\omega}_{r}\right)+\gamma \cdot \rho_{\text {glossy }}\left(\vec{\omega}_{i} \rightarrow \vec{\omega}_{r}\right),
$$

where $\gamma$ is a gloss factor.

The gloss factor is a real value between 0 and 1 representing how glossy a surface is. In this way totally diffuse, totally glossy, and hybrid surfaces can be modelled.

\subsection{Diffuse Reflection}

A perfectly diffuse surface will reflect all incident light isotropically and so appear equally bright from all directions. The Lambertian reflection model provides an accurate model for such surfaces. The apparent brightness of such a surface depends only upon the angle of incidence $\theta_{i}$ of incident light due to the projected surface area, hence Lambert's cosine law. Therefore, the BRDF for a given $\theta_{i}$ is constant. The reflectance function for Lambertian surfaces is 


$$
\rho_{\text {diffuse }}\left(\vec{\omega}_{i} \rightarrow \vec{\omega}_{r}\right)=1
$$

\subsection{Glossy Reflection}

The formulation of accurate models of glossy reflection has been the subject of much study in the domains of computer vision and image synthesis. One of the earliest, and simplest, models is due to Phong [9]. The reflectance function for Phong reflection is

$$
\rho_{\text {glossy }}\left(\vec{\omega}_{i} \rightarrow \vec{\omega}_{r}\right)=\cos ^{n}(\Phi),
$$

where $\Phi$ is the angular difference between the reflection direction $\left(\theta_{r}, \phi_{r}\right)$ and the plane mirror direction $\left(\theta_{i}, \phi_{i} \pm \pi\right)$, and $n$ is the specular-reflection exponent [3]. The specularreflection exponent is a measure of light scattering typically taking a value between 1 and 200. For a perfect reflector $n$ would be infinite.

Unlike the Lambertian model, the apparent surface brightness of a glossy surface is dependent upon the positions of both viewer and illuminant. For such a surface, maximum apparent brightness will occur when the camera lies in the plane mirror direction for a given illuminant position.

The Phong model is based on empirical estimation and is not a physical model of the reflection process. For this reason it does not provide accurate results for all types of surface material. It has been used in this paper for simplicity. Section 6 discusses how the model is to be improved for future work.

\section{Surface Model Estimation}

In this paper we attempt to determine the gloss factor of a surface by observing it over a period of time. During this interval the sun shall move over the surface being observed. It is expected that the apparent luminance of a surface, measured by image pixel luminance, will change as the sun moves across the sky hemisphere. The time of maximum pixel luminance will depend upon surface orientation, camera position, sun position and surface reflectance.

Surface radiance in the camera direction can be sampled throughout a time interval by taking a series of images of the scene. Each of these images is a single frame in a sequence spanning the time interval. The sun position for a particular frame of the sequence can be calculated from the scene location and local solar time [10], both of which shall be assumed known by the system. Due to atmospheric scattering (air mass) the direct insolation from the sun on a surface will also be dependent upon local solar time.

Figure 3 shows how reflected radiance from a planar surface in the camera direction varies across a sequence of nineteen frames. The graph shows reflected radiance values computed using Equation 2 for six different values of gloss factor. In each case the reflected radiance is computed by assuming the sun to be a point source of unit radiance and neglecting sky irradiance. Throughout the sequence both camera position and surface orientation are constant. The changing values of reflected radiance across the sequence are therefore determined by sun position. In this particular example frame 9 represents the local solar noon. It can be seen that maximum reflected radiance for each gloss factor occurs at a different point in the sequence. Ignoring the effects of air mass, the predicted time 
of maximum reflected radiance for a given gloss factor should correlate with the time of maximum pixel luminance for each surface.

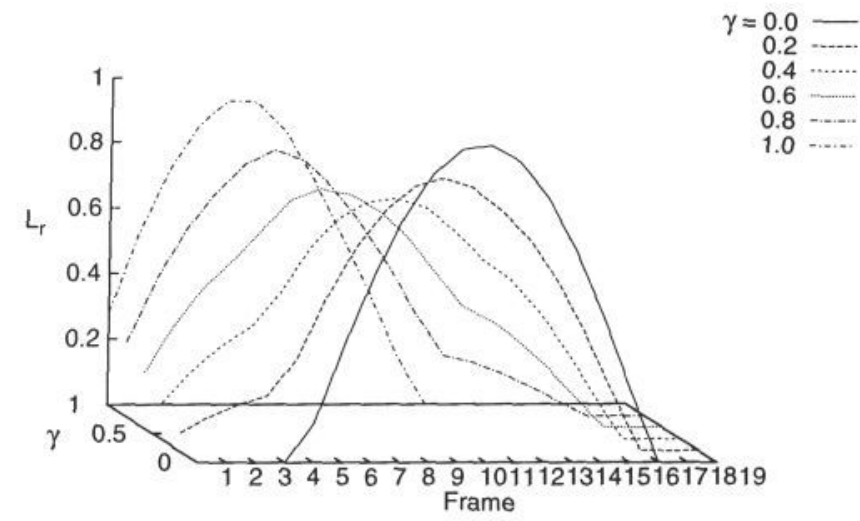

Figure 3: Surface radiance computed using Equation 2 across image sequence
for varying reflectance gloss factors.

The gloss factor for a surface can be estimated by comparing the observed brightness with that predicted by various reflectance models. This can be achieved by hypothesising gloss factors for a surface and correlating peaks in pixel luminance with the maximum reflected radiance predicted using each reflectance model. A good match will be when maximum surface radiance occurs within the same frame as maximum pixel luminance.

A limitation with this method arises from the nature of the Lambertian and Phong reflectance models given in Equations 5 and 6. If the sun's zenith position lies in the mirror direction with respect to the camera then both models will peak at the same time. In this situation it is expected that the system will perform poorly as the surface types become indistinguishable.

\section{Test Sequences}

System testing has been performed using synthetic image sequences. These have been generated using the Radiance lighting simulation package [11]. Radiance is a physically based image generation package and has been validated for use in such lighting simulation tasks $[5,6]$. The use of synthetic images allows full control over scene composition and illumination.

Radiance provides two basic material types; metal and plastic. These two materials can be modified using specularity and roughness values. Specularity, in this case, refers to light scattering by the surface material whereas roughness relates to perturbations of the surface normal. In our testing all surfaces were kept perfectly smooth with specularity values of $0.0,0.5$, and 1.0. For the purposes of this paper we shall denote surfaces with specularity $0.0,0.5$ and 1.0 as being matt, intermediate and glossy respectively .

Daylight simulation is supported in Radiance through the use of CIE standard sky models [2]. Two test skies have been used; the CIE standard clear and intermediate skies. No other light sources were included in the test scene. Overcast skies were not investigated 
due to the highly diffuse nature of the illumination. In such conditions only sky irradiance is incident on a surface and both matt and glossy surfaces would appear similar to our system.

The scene used throughout testing consisted of a single tessellated hemisphere placed on a flat ground plane. The origin of our local world coordinate system represented the location longitude $0^{\circ}$ and latitude $40^{\circ} \mathrm{N}$ on the Earth. The hemisphere was placed at the origin of this coordinate system. The scene could be viewed using a camera placed at five locations. These locations were; west, south-west, south, south-east, and east of the hemisphere.

For each material type and camera position a sequence of nineteen frames was generated. Each frame represented an image taken at hourly intervals starting at 04:00 and ending at 22:00. Figure 4 shows selected frames from a typical image sequence.
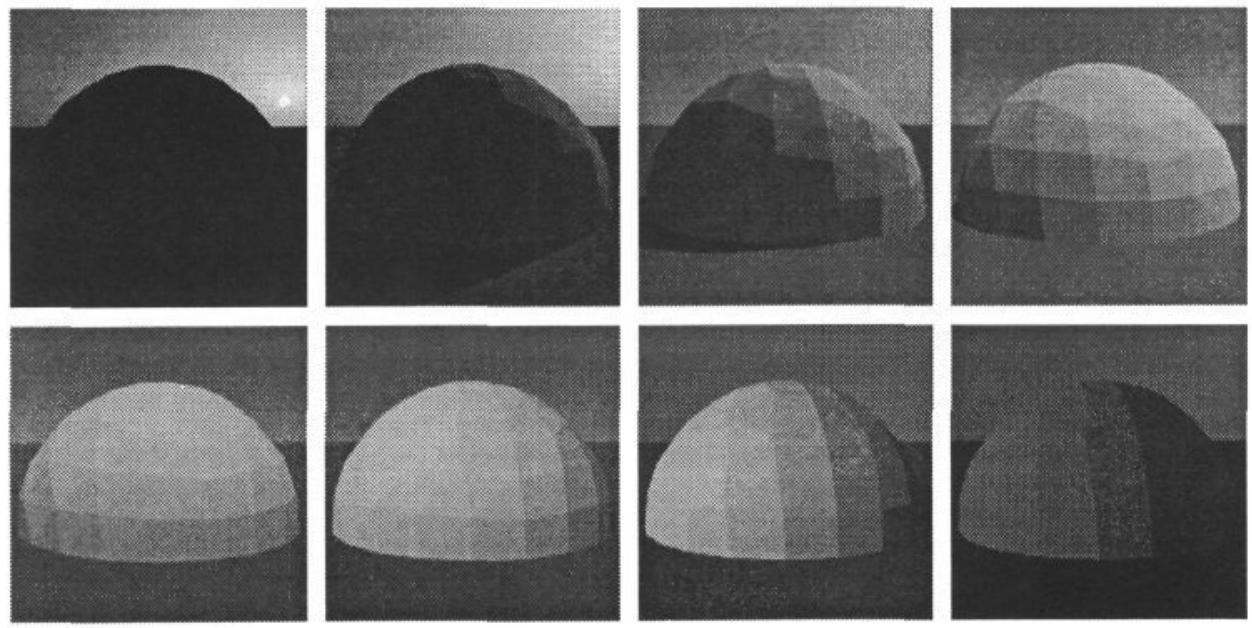

Figure 4: Typical image sequence viewing a matt hemisphere from the southwest. The first frame is taken at 05:00 with subsequent frames taken every two hours.

\section{Results}

In this section we address two aspects of our system's performance:

1. Correlation between maximum pixel luminance and peaks in reflected surface radiance predicted by our model for a single surface.

2. Estimation of gloss factor for a multi-faceted object.

\subsection{Single Surface Analysis}

Here we investigate the assumption made in Section 3 that peaks in both image pixel luminance and predicted surface radiance will occur in the same frame of an image sequence. 
This can be shown by comparing the observed luminance of a surface with various proposed models. Maximum pixel luminance for matt, intermediate and glossy surfaces should be predicted by using gloss factors of 0.0, 0.5 and 1.0 respectively in Equation 4.

Figure 5a shows sampled pixel luminance values for a surface made from matt, intermediate and glossy plastic when viewed from the west under a clear sky. Figure 5b shows the pixel values for the same surface illuminated by an intermediate sky. The associated reflected radiance computed using Equation 2 for the surface, based on gloss factors of $0.0,0.5$ and 1.0, is shown in Figure 5c

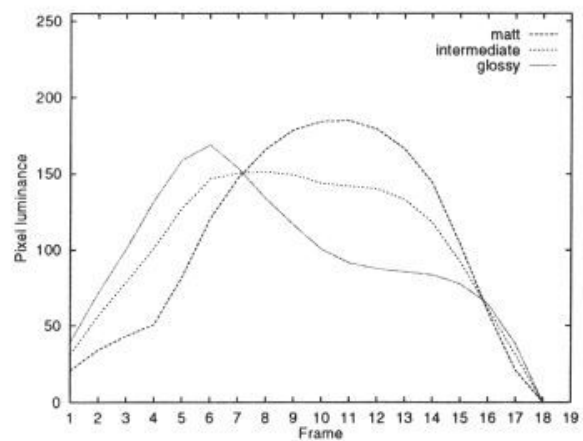

(a)

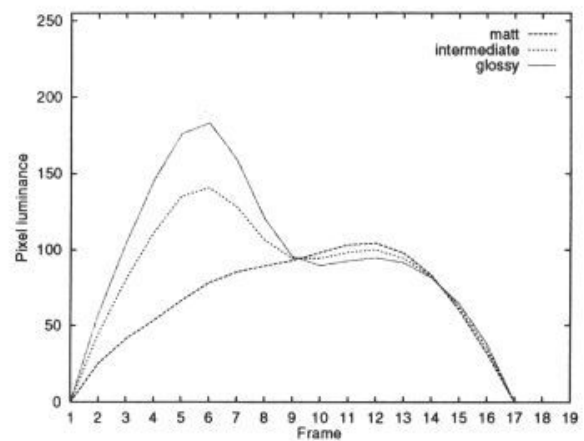

(b)

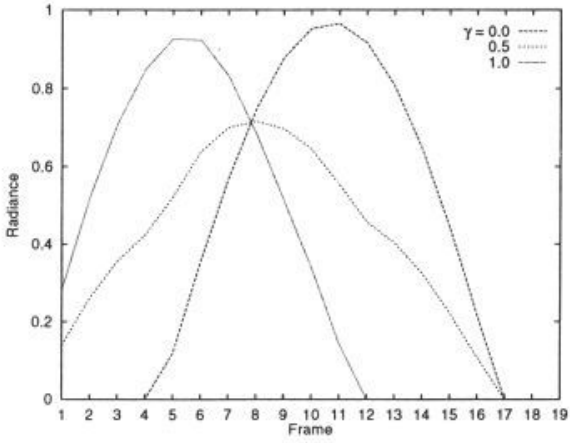

(c)

Figure 5: Pixel luminance and predicted reflected radiance for a surface made from matt, intermediate and glossy plastic viewed from the west; (a) Observations under clear sky illumination; (b) Observations under intermediate sky illumination; (c) Predicted surface radiance computed using reflectance models with gloss factors of 0.0, 0.5 and 1.0 in Equation 2.

It can be seen that under a clear sky, peak luminance for each surface type occurs at distinctly different times of the day. The position of these peaks correspond well with those of the predicted surface radiance computed using the expected reflectance model. This shows that maximum radiance predicted using a very simple reflectance model correlates well with the maximum luminance of a surface illuminated by a clear sky, despite differences in the shape of the luminance curves.

The results for surfaces viewed under an intermediate sky are less clear. It can be seen 
that the luminance peak for intermediate plastic has shifted to the same frame as that for glossy plastic, thus making the two materials indistinguishable using our simple reflection model. The shift in peak luminance between clear and intermediate skies is probably due to greater atmospheric scattering. This shows that our modelling of daylight illumination as a single point source is not suitable for intermediate type skies.

\subsection{Multi-Faceted Object Analysis}

We present here our results from estimating the gloss factor of a multi-faceted object. This testing was performed using the tessellated hemisphere sequences as described in Section 4. A total of 60 different sequences were used, each representing a different material, sky or viewing position. In each case the object's gloss factor was calculated by averaging the gloss factor for those surfaces which receive direct insolation. Each individual surface's gloss factor was calculated using the method described in Section 3. For matt, intermediate and glossy surfaces our method should ideally estimate gloss factors of $0.0,0.5$ and 1.0 respectively.

Figures $6 \mathrm{a}$ and $6 \mathrm{~b}$ show the estimated gloss factors for plastic and metallic hemispheres respectively. These two sets of results were collected from clear sky sequences. The results show that gloss factor estimation for matt and glossy objects is good, with little variance between views. The results for intermediate objects are, however, more varied. The system appears to overestimate gloss factor for plastic and underestimate it for metallic objects with an intermediate surface. This may well be due to differences in the metallic and plastic reflectance models of Radiance.

Results from sequences illuminated by intermediate skies are shown in Figures $6 \mathrm{c}$ and $6 \mathrm{~d}$. These results show a marked depreciation in performance for all types of surface. The accuracy of the system is now highly dependent on camera position, with results obtained from the south view having the greatest error. The poor results obtained from these sequences is probably due to the illumination being more diffuse. As for the single surface analysis of Section 5.1, the singular point source approximation of daylight would appear to be poor when dealing with intermediate skies.

\section{Future Developments}

This paper has shown that some crude estimation of surface gloss factor is possible using variations in sun position alone. More accurate results can be achieved by using improved illumination and reflection models. These will allow for better prediction of reflected radiance peaks and enable factors such as surface roughness to be taken into account.

The present system does not take into account irradiance from the sky or air mass. These factors significantly influence the shape of the sampled pixel luminance curves. As can be seen in Figure 5 luminance values sampled from specular surfaces have a characteristically different shape to those from matt surfaces. This is because the matt surface is reflecting light towards the camera from all directions, not just from the sun. It is hoped that the inclusion of sky irradiance will enable the analysis of surfaces that do not receive direct insolation from the sun.

It is also our intention to use more physically plausible reflection models. The work presented here highlights the limitations of using only a simple local reflection model. 


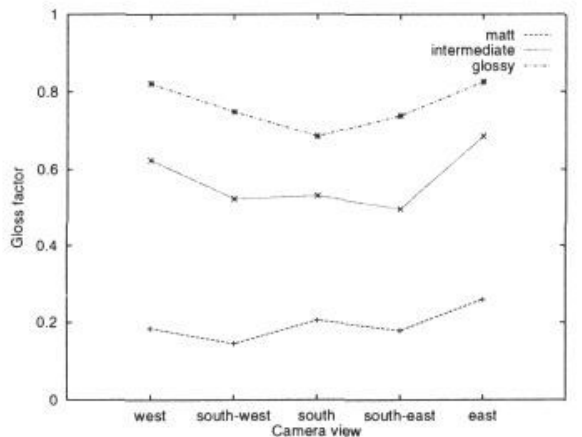

(a)

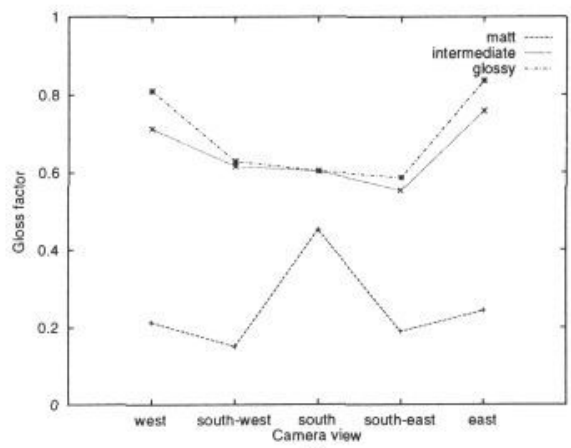

(c)

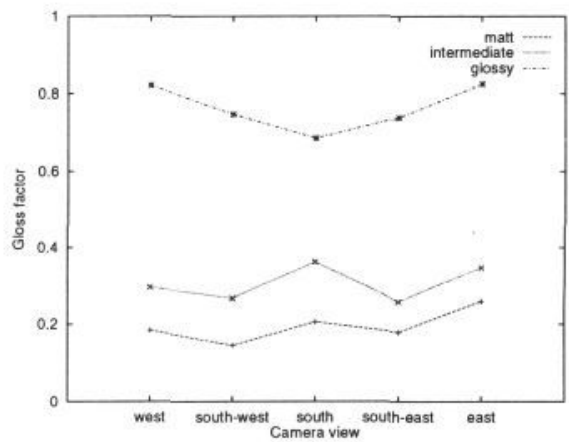

(b)

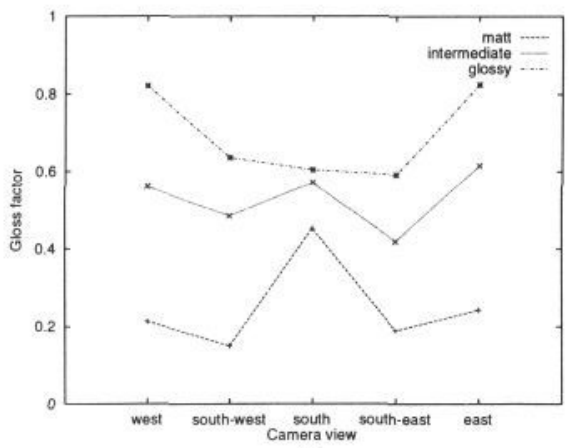

(d)

Figure 6: Results for matt, intermediate and glossy hemispheres; (a) plastic hemisphere, clear sky; (b) metallic hemisphere, clear sky; (c) plastic hemisphere, intermediate sky; (d) metallic hemisphere, intermediate sky.

The Phong model used as the glossy component in Equation 4 is a poor approximation for metallic surfaces and is to be replaced.

There are drawbacks to the use of synthetic image sequences for system testing. Synthetic image generation inevitably involves simplification: care must be taken so that we do not over simplify the situation and hence render the results meaningless. It is, however, necessary to test systems using scenes with known illuminants and composition, without the problems of ensuring consistent conditions and linear camera response. By using synthetic data we have a controlled environment in which we can vary parameters independently and study their influence on system performance. Work in progress is exploring the sensitivity of the system to factors such as; image noise, camera linearity, range and sparsity of illumination, viewing and illumination geometry, and choice of reflection model. These simulations will be used to guide interpretation of the results when the system is applied to real scenes. 


\section{Conclusions}

We have shown that by using simple models of reflection and daylight illumination we can determine, with varying degrees of accuracy, the gloss factor of a naturally illuminated surface. The accuracy of the system is largely dependent upon the sky conditions under which the scene is viewed. Owing to the simplistic reflection models and method used, some instances of sun position and viewing geometry make it difficult to determine surface gloss.

It is hoped that the enhancements described in Section 6 will allow more accurate results to be obtained from real image sequences, regardless of viewing geometry.

The results presented in this paper have nevertheless shown that complicated illumination, such as daylight, can be modelled simplistically for machine vision purposes. The results obtained from using such methods could be used for deeper levels of analysis in a hierarchical vision system.

\section{References}

[1] M. Cohen, J. Wallace, and P. Hanrahan. Radiosity and Realistic Image Synthesis. Academic Press, 1993.

[2] Commision International de l'Éclairage. Spatial distribution of daylight - Luminance distribution of various reference skies, CIE Publication 110, 1994.

[3] J. D. Foley, A. van Dam, S. K. Feiner, and J. F. Hughes. Computer Graphics: Principles and Practice. Addison-Wesley, second edition, 1990.

[4] A. Gagalowicz. Tools for advanced telepresence systems. Computers and Graphics, 19(1):73-88, 1995.

[5] A. Grynberg. Validation of radiance. Technical Report LBID 1575, Lawrence Berkley Laboratory, July 1989.

[6] J. Mardaljevic, K. L. Lomas, and D. G. Henderson. Advanced daylighting design for complex spaces. In Proceedings of CLIMA 2000, London UK, November 1993.

[7] S. K. Nayer, K. Ikeuchi, and T. Kanade. Determining shape and reflectance of hybrid surfaces by photometric sampling. IEEE Trans. on Robotics and Automation, 6(4):418-431, August 1990.

[8] F. E. Nicodemus, J. C. Richmond, and J. J. Hsia. Geometrical considerations and nomenclature for reflectance. National Bureau of Standards Monograph 160, 1977.

[9] B. T. Phong. Illumination in computer generated pictures. Communications of the ACM, 18(8):311-317, 1975 .

[10] D. Rapp. Solar Energy. Prentice-Hall, 1981.

[11] G. J. Ward. The RADIANCE lighting simulation and rendering system. In SIGGRAPH '94 Proceedings, 1994. 